\title{
Bromate-1,4-Cyclohexanedione-Ferroin Gas-Free Oscillating Reaction. 1. Basic Features and Crossing Wave Patterns in a Reaction-Diffusion System without Gel
}

\author{
Krisztina Kurin-Csörgei, ${ }^{\dagger}$ Anatol M. Zhabotinsky, ${ }^{*}, \stackrel{\star}{ }$ Miklós Orbán, ${ }^{*, \dagger}$ and Irving R. Epstein \\ Department of Chemistry and Volen Center for Complex Systems, Brandeis University, \\ Waltham, Massachusetts 02254-9110, and Institute for Inorganic and Analytical Chemistry, \\ L. Eötvös University, P.O. Box 32, Budapest 112, Hungary
}

Received: November 14, $1995^{\otimes}$

\begin{abstract}
We present a study of the bromate-1,4-cyclohexanedione-ferroin oscillating reaction in both well-stirred and reaction-diffusion systems. We investigate the dependence of the system dynamics on the initial reagent concentrations, with the initial concentration of ferroin varied from 0 to $5 \times 10^{-3} \mathrm{M}$, and show that the system behaves like a typical BZ oscillator if the concentration of ferroin exceeds $5 \times 10^{-5} \mathrm{M}$. We demonstrate spontaneous formation of spiral waves and transverse wave fronts in quasi-two-dimensional systems and formation of crossing wave patterns in thin layers of solution open to air.
\end{abstract}

\section{Introduction}

The Belousov-Zhabotinsky (BZ) reaction-diffusion system is perhaps the most commonly employed tool for experiments on chemical waves and pattern formation. ${ }^{1-7}$ The ferroincatalyzed reaction with malonic acid as the initial reductant has been the most frequently used BZ system for this purpose. An important drawback of this reaction is the production of carbon dioxide, which results in the formation of bubbles that disturb the reaction-diffusion patterns and make it difficult to conduct lengthy experiments in either batch or open reactors. Attempts have been made to develop gas-free versions of the $\mathrm{BZ}$ reaction. ${ }^{8}$ Unfortunately, these variants do not generate the range of chemical wave behavior found in the ferroin-malonic acid BZ reaction. Recently, Kurin-Csörgei et al. ${ }^{9}$ showed that target patterns develop in the bromate-1,4-cyclohexanedione-ferroin reaction-diffusion system without formation of bubbles. They used ferroin at a concentration of $5 \times 10^{-4} \mathrm{M}$ and considered it to be only an indicator, implying that the reaction is an uncatalyzed bromate oscillator (UBO). ${ }^{9-12}$

Here we present further results related to both the uncatalyzed and the ferroin-catalyzed systems. We study the dependence of the system dynamics on the initial reagent concentrations, with that of ferroin varied from 0 to $5 \times 10^{-3} \mathrm{M}$, and show that the system behaves like a typical BZ oscillator if the concentration of ferroin exceeds $5 \times 10^{-5} \mathrm{M}$.

The absence of bubbles and precipitate makes the present system an excellent tool for experiments on chemical waves and patterns. We demonstrate spontaneous formation of spiral waves and transverse wave fronts in quasi-two-dimensional systems and the formation of crossing wave patterns in thin layers of solution open to air.

Some preliminary mechanistic considerations are also suggested.

\section{Experimental Section}

Materials. Stock solutions of $1.0 \mathrm{M} \mathrm{NaBrO}$ (Janssen Chimica, 99+\%), $0.3 \mathrm{M}$ 1,4-cyclohexanedione (abbreviated as CHD) (Aldrich, 98\%) dissolved in $1.0 \mathrm{M} \mathrm{H}_{2} \mathrm{SO}_{4}$ by stirring the solution on a hot plate $\left(40-50{ }^{\circ} \mathrm{C}\right.$ ) for about $30 \mathrm{~min}, 0.025 \mathrm{M}$

\footnotetext{
L. Eötvös University.

* Brandeis University.

${ }^{\otimes}$ Abstract published in Advance ACS Abstracts, March 1, 1996.
}

ferroin (prepared from the calculated amount of $\mathrm{FeSO}_{4} \cdot 7 \mathrm{H}_{2} \mathrm{O}$ (Fisher, certified) and 1,10-phenanthroline (Aldrich, 99+\%)), $0.2 \mathrm{M} \mathrm{NaBr}$ (Fisher, certified), and 1.0-10.0 $\mathrm{M} \mathrm{H}_{2} \mathrm{SO}_{4}$ were used to prepare the working solutions.

Apparatus and Procedures. Batch experiments with the bromate-CHD and the bromate-CHD-ferroin systems were performed in a thermostated $\left(T=25\right.$ or $\left.35^{\circ} \mathrm{C}\right)$ vessel equipped with a PT $v s \mathrm{Hg}\left|\mathrm{Hg}_{2} \mathrm{SO}_{4}\right| \mathrm{K}_{2} \mathrm{SO}_{4}$ electrode pair, and the potential of the Pt electrode was monitored with a chart recorder.

The kinetics of the reaction between ferriin and CHD were measured by recording the decrease of the absorbance of ferriin at $\lambda=626 \mathrm{~nm} v$ s time using a diode-array spectrophotometer.

In some experiments, mixtures of bromate, $\mathrm{CHD}$, and $\mathrm{H}_{2} \mathrm{SO}_{4}$ were used. Under appropriate conditions such mixtures can oscillate in batch and probably produce patterns in thin unstirred layers similar to those found in other uncatalyzed bromate oscillators. ${ }^{13}$ Oscillations in redox potential appear after a long induction period (5-90 $\mathrm{min})$, and the pattern, if it exists, cannot be seen by eye, because no colored intermediates are formed. To overcome these shortcomings, bromide ions - to shorten or eliminate the induction period-and ferroin indicator-to make the oscillations and the pattern formation visible-were also introduced.

For studying wave formation and propagation in the bromate-CHD-ferroin system, the mixture was spread in a thin layer in either a glass or a plastic petri dish $(10,7$, or $6 \mathrm{~cm}$ i.d.) or between a pair of glass plates. The thickness of the layer varied between 0.5 and $2.0 \mathrm{~mm}$. In the uncovered petri dish, the system was exposed to air, creating gradients in the concentrations of oxygen and of bromine, which evolves during the course of the reaction. When the solution layer was placed between glass plates, oxygen was excluded from, the bromine was trapped in, the system. In these experiments, we employed solution layers with thicknesses of 0.8 and $1.2 \mathrm{~mm}$. The sides of the layers were isolated from the atmosphere by plastic spacers.

The evolution of the wave patterns was recorded with a video camera augmented with an interference filter with maximum transmission at $510 \mathrm{~nm}$. The data were processed with an OPTIMAS image-analysis system (BioScan).

\section{Results}

Induction Period. A rather long induction period occurs prior to the beginning of oscillations. In the uncatalyzed system 

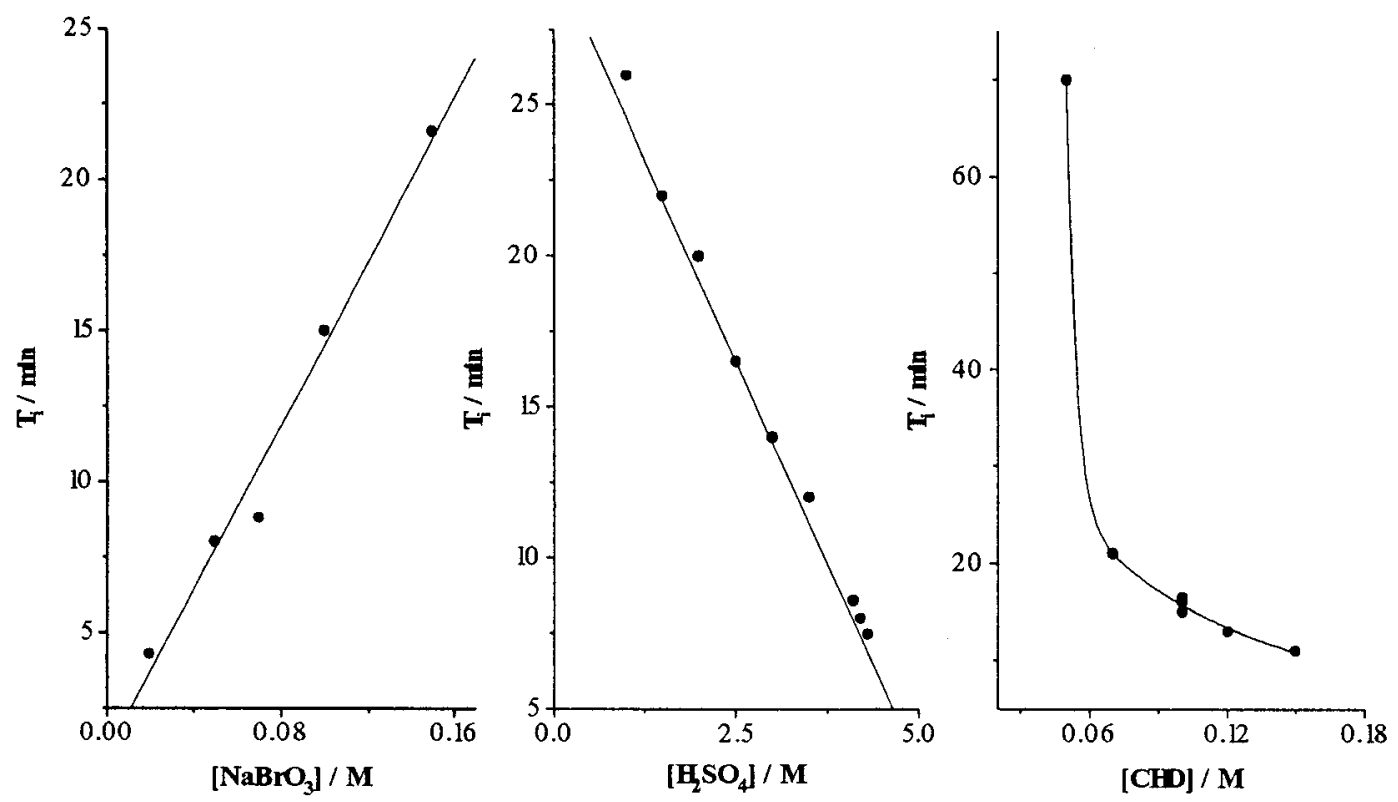

Figure 1. Dependence of the induction period $\left(T_{\mathrm{i}}\right)$ on the initial concentrations of bromate, sulfuric acid, and $\mathrm{CHD}$. $T=35^{\circ} \mathrm{C}$. Initial concentrations (in M): (a) $\mathrm{H}_{2} \mathrm{SO}_{4} 2.5$, CHD 0.1; (b) $\mathrm{NaBrO}_{3} 0.1$, CHD 0.1; (c) $\mathrm{H}_{2} \mathrm{SO}_{4} 2.5, \mathrm{NaBrO}_{3} 0.1$.
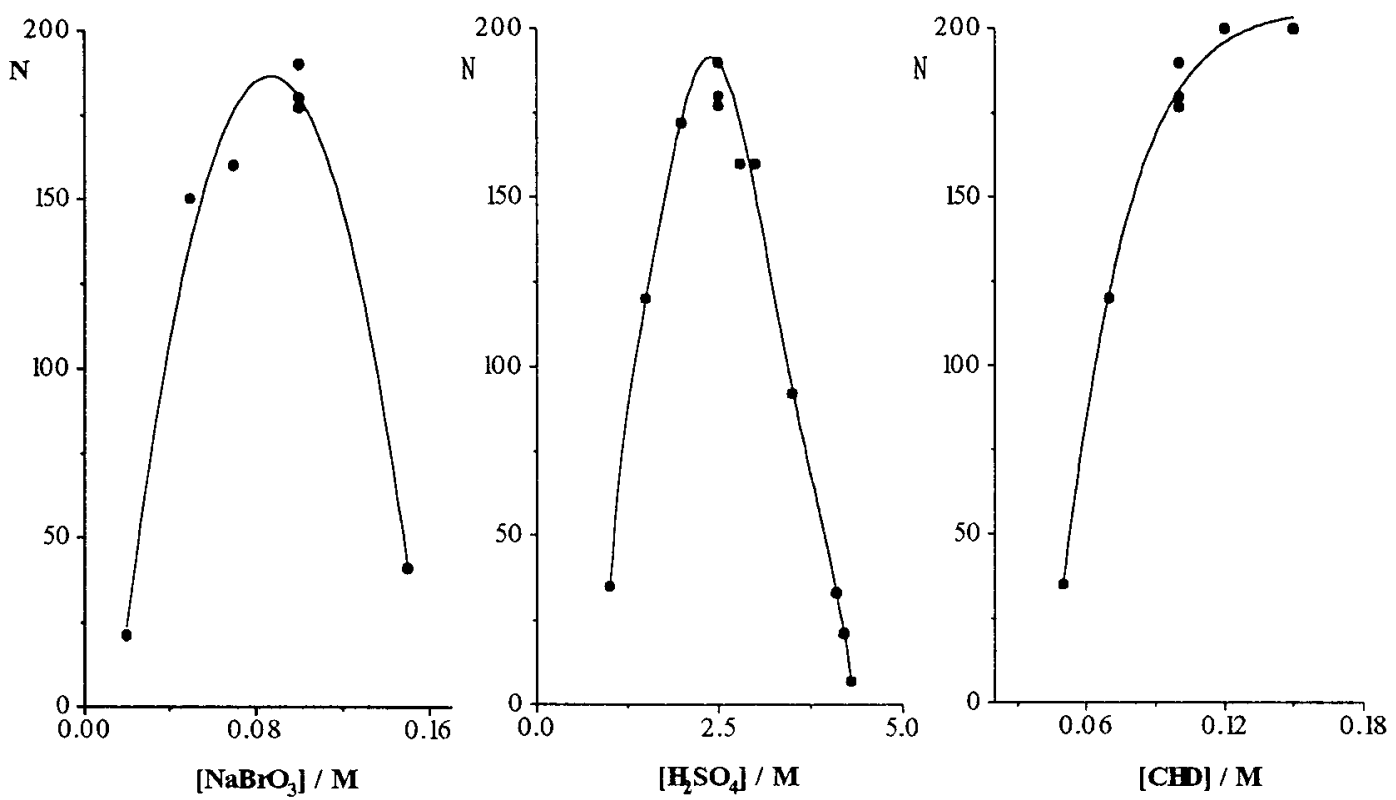

Figure 2. Dependence of the number of oscillations on the initial concentrations of bromate, sulfuric acid, and CHD. $T=35^{\circ} \mathrm{C}$. Initial concentrations (in M): (a) $\mathrm{H}_{2} \mathrm{SO}_{4} 2.5$, CHD 0.1; (b) $\mathrm{NaBrO}_{3} 0.1$, CHD 0.1; (c) $\mathrm{H}_{2} \mathrm{SO}_{4} 2.5 ; \mathrm{NaBrO}_{3} 0.1$.

the duration of the induction period $T_{\mathrm{i}}$ increases linearly with increasing $\left[\mathrm{NaBrO}_{3}\right]$, decreases linearly with increasing $\left[\mathrm{H}_{2} \mathrm{SO}_{4}\right]$, and decreases with increasing [CHD]. Figure 1 shows $T_{\mathrm{i}}$ as function of the initial reagent concentrations.

Partial bromination of CHD significantly decreases $T_{\mathrm{i}}$. The bromination occurs via the following reaction in aqueous solution:

$$
3 \mathrm{H}^{+}+\mathrm{BrO}_{3}^{-}+2 \mathrm{Br}^{-}+3 \mathrm{CHD} \rightarrow 3 \mathrm{BrCHD}+3 \mathrm{H}_{2} \mathrm{O}
$$

For example, the induction period is equal to $68 \mathrm{~min}$ at $25^{\circ} \mathrm{C}$ when the initial concentrations are $\left[\mathrm{NaBrO}_{3}\right]=0.1 \mathrm{M}$, [CHD] $=0.1 \mathrm{M}$, and $\left[\mathrm{H}_{2} \mathrm{SO}_{4}\right]=1.0 \mathrm{M}$. Adding $\mathrm{NaBr}$ in concentrations $0.01 \mathrm{M}$ and 0.02 reduces the induction period to 51 and 0.4 min, respectively. Ferroin, in concentrations up to $5 \times 10^{-3}$ M, does not significantly affect the induction period.

Dependence of Batch Oscillations on the Initial Reagent Concentrations. Oscillations in the uncatalyzed system have been found in the following range of initial reagent concentrations: $\mathrm{NaBrO}_{3}=0.02-0.15 \mathrm{M}, \mathrm{CHD}=0.05-0.15 \mathrm{M}$, and $\mathrm{H}_{2} \mathrm{SO}_{4}=0.5-4.3 \mathrm{M}$.

The oscillations begin after the induction period and last from 20 to $60 \mathrm{~min}$. The amplitude of oscillation of the redox potential gradually increases from $2-4 \mathrm{mV}$ up to about $100 \mathrm{mV}$. The period is generally in the range $3-10 \mathrm{~s}$. Lower acid concentration results in larger amplitude, longer period oscillations, and a longer induction period. Figure 2 shows the number of cycles of oscillation in the uncatayzed system as a function of the initial reagent concentrations.

Effect of Ferroin. Adding ferroin strongly affects the batch oscillations: the period of oscillations and the number of cycles increase by as much as a factor of 5, depending on the ferroin concentration. Figure 3 shows the dependence of the oscillation period $\left(T_{\mathrm{o}}\right)$ on the ferroin concentration. One can see that with concentrations of ferroin $\leq 5 \times 10^{-5} \mathrm{M}$ the oscillation period does not change within our precision. The number of cycles 


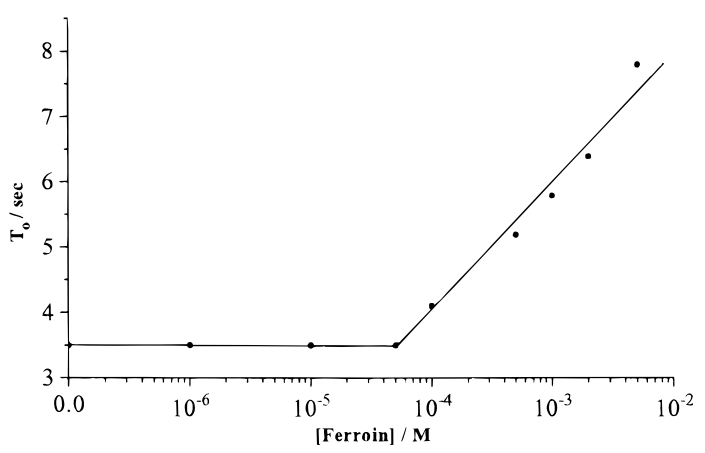

Figure 3. Dependence of the oscillation period $\left(T_{\mathrm{o}}\right)$ on the initial concentration of ferroin. $T_{\mathrm{o}}$ is the mean value of the oscillation period for the first $5 \mathrm{~min}$ after the start of oscillation. $T=25^{\circ} \mathrm{C}$. Initial concentrations (in $\mathrm{M}$ ): $\mathrm{NaBrO}_{3} 0.1, \mathrm{CHD} 0.1, \mathrm{H}_{2} \mathrm{SO}_{4} 2.5, \mathrm{KBr} 0.01$.

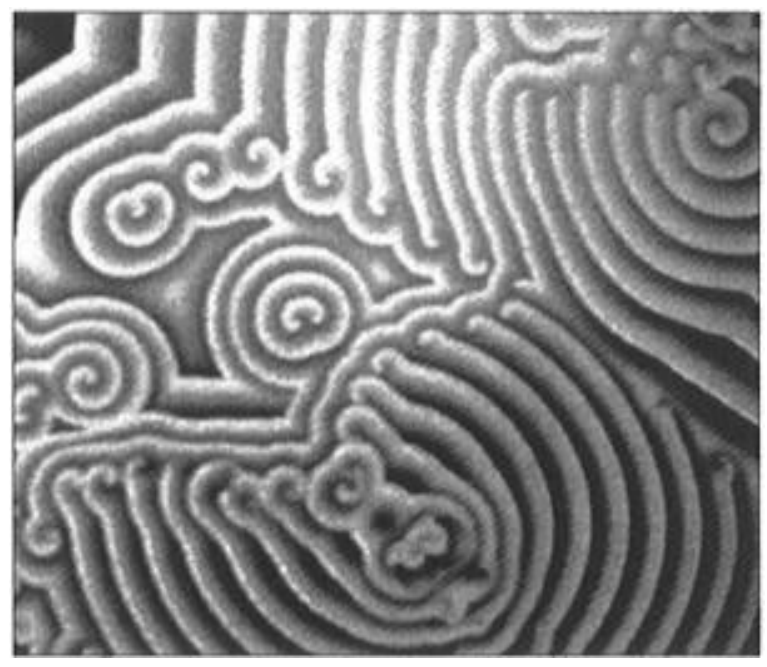

Figure 4. Formation of waves transverse to the initial wave trains through restricted spiral formation in a solution layer trapped between two glass plates. $T=25^{\circ} \mathrm{C}$. Initial concentrations (in $\mathrm{M}$ ): $\mathrm{NaBrO}_{3}$ $0.1, \mathrm{CHD} 0.1, \mathrm{H}_{2} \mathrm{SO}_{4} 0.2, \mathrm{KBr} 0.01, \mathrm{Fe}(\mathrm{phen})_{3}{ }^{2+} 0.005$. Frame size $(\mathrm{mm}): 62 \times 54$.

and the amplitude of oscillation of the redox potential also remain practically unchanged at low ferroin concentrations. $T_{\mathrm{o}}$ rises approximately linearly with increasing ferroin concentration above $1 \times 10^{-4} \mathrm{M}$. The number of cycles and amplitude of redox potential oscillations also rise in approximately the same manner.

In the ferroin-catalyzed reaction, oscillations take place at concentrations of $\mathrm{H}_{2} \mathrm{SO}_{4}$ as low as $0.1 \mathrm{M}$, while in the uncatalyzed system we were not able to obtain oscillations below $0.5 \mathrm{M}$.

Reduction of Ferriin by CHD. We employ the initial concentrations $\mathrm{Fe}$ (phen) $3^{3+}=5 \times 10^{-4} \mathrm{M}, \mathrm{CHD}=0.1 \mathrm{M}$, and $\mathrm{H}_{2} \mathrm{SO}_{4}=0.2 \mathrm{M}$, or $1.03 \mathrm{M}$, to evaluate the kinetics of ferriin reduction by $\mathrm{CHD}$. The decay of $\left[\mathrm{Fe}(\text { phen })_{3}{ }^{3+}\right]$ is exponential, and the corresponding second-order rate constant is $0.23 \pm 0.01$ $\mathrm{M}^{-1} \mathrm{~s}^{-1}$ at $\left[\mathrm{H}_{2} \mathrm{SO}_{4}\right]=0.2 \mathrm{M}$ and $0.360 \pm 0.004 \mathrm{M}^{-1} \mathrm{~s}^{-1}$ at $\left[\mathrm{H}_{2} \mathrm{SO}_{4}\right]=1.03 \mathrm{M}$.

Patterns in Monophasic Layers. We obtained the familiar $\operatorname{target}^{1,2}$ and spiral wave ${ }^{2,3}$ patterns in solution layers placed between two glass plates. The patterns persist for several hours in batch systems. Increasing the ferroin concentration results in better contrast but reduces the lifetime of the patterns.

We observed spontaneous formation of waves transverse to the initial wave trains (Figure 4). Several routes lead to the formation of transverse waves; we shall describe the details of their evolution in a subsequent publication. In many cases, a flank of a wave train is temporarily confined by a nonexcitable

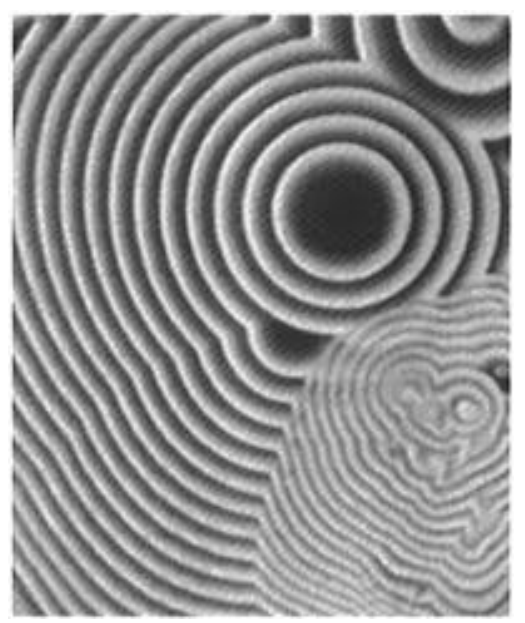

Figure 5. Smooth crossing waves in a solution layer open to air. Conditions are as in Figure 4. Frame size $(\mathrm{mm}): 58 \times 70$.

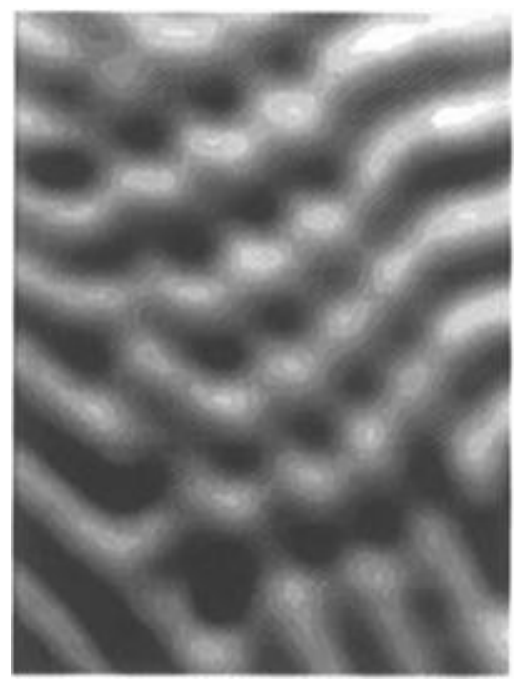

Figure 6. Sawtooth crossing waves in a solution layer open to air. Conditions are as in Figure 4. Frame size $(\mathrm{mm}): 10 \times 13$.

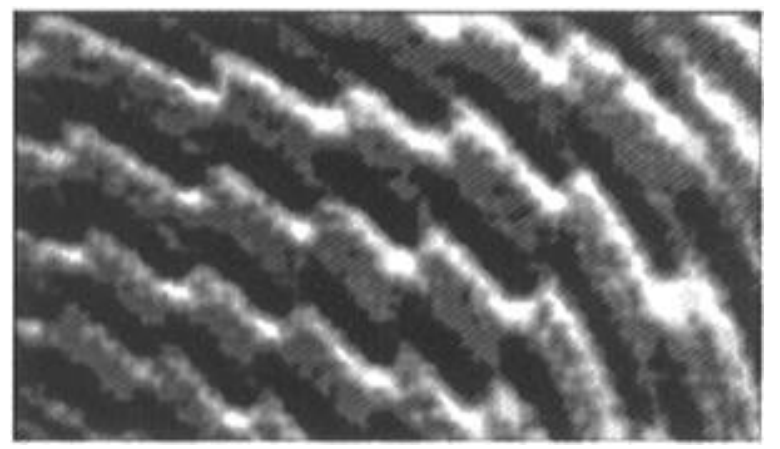

Figure 7. Staircase crossing waves in a solution layer open to air. Conditions are as in Figure 4. Frame size $(\mathrm{mm}): 17.5 \times 10$.

region. When this restraint disappears, the free ends of the wave fronts start to form spirals. After the spiral parts of the waves collide with the next wave fronts in the train, they fuse, forming a curved wave front transverse to the initial wave fronts. Finally, this transverse wave front flattens due to the curvature effect. ${ }^{14}$ When the refractory region, which follows the transverse front, moves away from the flank of the initial wave train, a new cycle of spiral formation begins, which eventually gives rise to a second transverse wave front, and so on.

Crossing Wave Patterns. In layers exposed to air, we have observed spontaneous formation of all types of crossing wave (CW) patterns found previously in gel layers, ${ }^{15,16}$ but which we 
have been unable to reproduce in the gel-free bromate-ferroinmalonic acid system. Figure 5 shows a smooth crossing wave pattern. Figure 6 presents a sawtooth $\mathrm{CW}$ pattern, and Figure 7 shows a staircase $\mathrm{CW}$ pattern.

We have shown ${ }^{17}$ that crossing wave patterns can be obtained with various types of gels and have argued that the gel is needed only to prevent microconvection. Our observations in the CHD system support this contention.

\section{Discussion}

Several studies of the mechanism of the $\mathrm{UBO}^{12,18,19}$ propose that the main source of bromide production is the reduction of $\mathrm{HOBr}$ by organic free radicals. These mechanisms neglect bromoderivatives of the initial reductant as a source of bromide, despite the fact that the oxidation of these bromoderivatives is believed to deliver most of the $\mathrm{Br}^{-}$in the catalyzed $\mathrm{BZ}$ reaction. An alternative mechanism for the $\mathrm{UBO}^{20}$ suggests that bromoderivatives of the organic substrate provide the source of $\mathrm{Br}^{-}$. Our experiments show that prebromination of CHD diminishes, and can practically eliminate, the induction period. This observation lends support to the assumption that brominated CHD serves as the sole source of $\mathrm{Br}^{-}$in a skeleton scheme of the CHD oscillator. Organic compounds like CHD are known to undergo aromatization during oxidation, yielding hydroquinone $\left(\mathrm{H}_{2} \mathrm{Q}\right)$ and finally quinone $(\mathrm{Q})$ as products. ${ }^{21-23}$

A simple skeleton scheme for the uncatalyzed CHD oscillator is

$$
\begin{aligned}
& \mathrm{H}^{+}+\mathrm{Br}^{-}+\mathrm{HOBr} \rightleftarrows \mathrm{Br}_{2}+\mathrm{H}_{2} \mathrm{O} \\
& \mathrm{H}^{+}+\mathrm{Br}^{-}+\mathrm{HBrO}_{2} \rightleftarrows 2 \mathrm{HOBr} \\
& \mathrm{H}^{+}+\mathrm{Br}^{-}+\mathrm{HBrO}_{3} \rightleftarrows \mathrm{HBrO}_{2}+\mathrm{HOBr} \\
& 2 \mathrm{HBrO}_{2} \rightleftarrows \mathrm{HOBr}+\mathrm{HBrO}_{3} \\
& \mathrm{H}^{+}+\mathrm{BrO}_{3}^{-} \rightleftarrows \mathrm{HBrO}_{3} \\
& \mathrm{H}^{+}+\mathrm{HBrO}_{3}+\mathrm{HBrO}_{2} \rightleftarrows \mathrm{HBrO}_{2}^{+}+\mathrm{BrO}_{2}^{\bullet}+\mathrm{H}_{2} \mathrm{O} \\
& \mathrm{BrO}_{2}^{\bullet}+\mathrm{H}^{+} \rightleftarrows \mathrm{HBrO}_{2}^{+} \\
& \mathrm{BrO}_{2}^{\bullet}+\mathrm{CHD} \rightleftarrows \mathrm{HBrO}_{2}+\mathrm{CHD}^{\bullet} \\
& 2 \mathrm{CHD}^{\bullet} \rightarrow \mathrm{CHD}+\mathrm{H}_{2} \mathrm{Q} \\
& \mathrm{CHD}+\mathrm{HOBr} \rightarrow \mathrm{BrCHD}+\mathrm{H}_{2} \mathrm{O} \\
& \mathrm{CHD}^{\bullet}+\mathrm{BrCHD} \rightarrow \mathrm{CHD}+\mathrm{BrCHD}^{\bullet} \\
& \mathrm{CHD}^{\bullet}+\mathrm{BrCHD}^{\bullet} \rightarrow 2 \mathrm{H}_{2} \mathrm{Q}+\mathrm{H}^{+}+\mathrm{Br}^{-}
\end{aligned}
$$

This scheme neglects reactions of hydroquinone.

An alternative simple scheme can be obtained by substituting reactions $\mathrm{R} 11-\mathrm{R} 15$ for reactions $\mathrm{R} 6, \mathrm{R} 7, \mathrm{R} 9$, and R10:

$$
\begin{gathered}
\mathrm{HBrO}_{3}+\mathrm{CHD} \rightarrow \mathrm{H}_{2} \mathrm{Q}+\mathrm{HBrO}_{2}+\mathrm{H}_{2} \mathrm{O} \\
\mathrm{BrO}_{2}^{\bullet}+\mathrm{H}_{2} \mathrm{Q} \rightleftarrows \mathrm{HBrO}_{2}+\mathrm{HQ}^{\bullet} \\
2 \mathrm{HQ}^{\bullet} \rightarrow \mathrm{H}_{2} \mathrm{Q}+\mathrm{Q}
\end{gathered}
$$

$$
\begin{gathered}
\mathrm{HQ}^{\bullet}+\mathrm{BrCHD} \rightarrow \mathrm{HQ}+\mathrm{BrCHD}^{\bullet} \\
\mathrm{HQ}^{\bullet}+\mathrm{BrCHD}^{\bullet} \rightarrow \mathrm{H}_{2} \mathrm{Q}+\mathrm{Q}+\mathrm{H}^{+}+\mathrm{Br}^{-}
\end{gathered}
$$

Here $\mathrm{HQ}^{\bullet}$ is semiquinone. In reality, all the above reactions can take place, together with many others.

In the presence of ferroin the following reactions must be included:

$$
\begin{gathered}
\mathrm{Fe}(\text { phen })_{3}^{2+}+\mathrm{HBrO}_{2}^{+} \rightleftarrows \mathrm{Fe}(\text { phen })_{3}^{3+}+\mathrm{HBrO}_{2} \\
\mathrm{Fe}(\text { phen })_{3}^{3+}+\mathrm{CHD} \rightleftarrows \mathrm{Fe}(\text { phen })_{3}^{2+}+\mathrm{CHD}^{\bullet}+\mathrm{H}^{+} \\
\mathrm{Fe}(\text { phen })_{3}^{3+}+\mathrm{H}_{2} \mathrm{Q} \rightleftarrows \mathrm{Fe}(\text { phen })_{3}^{2+}+\mathrm{HQ}^{\bullet}+\mathrm{H}^{+}
\end{gathered}
$$

Direct oxidation of BrCHD by ferriin can also contribute to the production of $\mathrm{Br}^{-}$.

The uncatalyzed reaction does not generate oscillations at low concentrations of sulfuric acid. In this case the simplest scheme for the bromate-CHD-ferroin oscillator consists of reactions $\mathrm{R} 1-\mathrm{R} 5$, R6a, and R8-R10. It is worth noting that the rate constant for the reduction of ferriin by CHD is close to that for the reduction of cerium(IV) by malonic acid. We may therefore expect that this scheme will generate oscillations resembling those in the cerium-malonic acid BZ reaction.

We intend to study kinetic models based on reactions R1R17 and to compare the results of simulations with our experimental data to clarify the mechanism of the bromateCHD oscillator.

In BZ layers open to air, a nonmonotonic gradient of excitability can emerge as a result of two opposite concentration gradients: those of oxygen and of bromoderivatives of the initial reductant. ${ }^{17}$ We suggest that the same mechanism is involved in the present system. The excitability profile depends strongly on the layer thickness. In BZ systems small variations of the layer thickness can generate crossing wave patterns. ${ }^{24}$ In open liquid layers these variations can easily result from menisci which form as a result of the surface tension.

The absence of $\mathrm{CO}_{2}$ production is a great advantage of the CHD system. Without $\mathrm{CO}_{2}$ bubbles it is easy to prevent mechanical perturbation of the solution. As a result, we have been able to observe chemical wave patterns which had previously been seen only in gel systems. Gel-free systems are often more convenient for experiments and are more uniform than gel systems. The absence of bubbles also facilitates operation of continuously fed unstirred reactors (CFUR). For these reasons, the $\mathrm{BZ}$ reaction with 1,4-cyclohexanedione as the reductant should be a valuable tool for the study of chemical waves and patterns.

Acknowledgment. This work was supported by the National Science Foundation (Grants CHE-9304087 and CHE-9023294), by a U.S.-Hungarian cooperative grant from the NSF (INT9322738), and by the Hungarian Academy of Sciences (OTKA T 016680 and F 017073). We thank Matthew Eager and István Szalai, for help in performing kinetics and some batch experiments, and Milos Dolnik for a critical reading of the manuscript.

\section{References and Notes}

(1) Zaikin, A. N.; Zhabotinsky, A. M. Nature 1970, 225, 535.

(2) Zhabotinsky, A. M.; Zaikin, A. N. In Oscillating Processes in Biological and Chemical Systems II; Sel'kov, E. E., Ed.; Nauka: Puschino; 1971; p 279

(3) Winfree, A. T. Science 1972, 175, 634.

(4) Field, R. J., Burger, M., Eds. Oscillations and Traveling Waves in Chemical Systems; Wiley: New York, 1985.

(5) Ross, J.; Müller, S. C.; Vidal, C. Science 1988, 240, 460. 
(6) Swinney, H. L., Krinsky, V. I., Eds. Waves and Patterns in Chemical and Biological Media; Physica 1991, $49 D(1 / 2)$.

(7) Kapral, R., Showalter, K., Eds. Chemical Waves and Patterns; Kluwer: Dordrecht, 1995.

(8) Ouyang, Q.; Tam, W. Y.; De Kepper, P.; McCormick, W. D.; Noszticzius, Z.; Swinney, H. L. J. Phys. Chem. 1987, 91, 2181.

(9) Kurin-Csörgei, K.; Szalai, I.; Körös, E. React. Kinet. Catal. Lett. 1995, 54, 217.

(10) Kurin-Csörgei, K.; Szalai, I.; Molnár-Perl, I.; Körös, E. React. Kinet. Catal. Lett. 1995, 53, 115.

(11) Orbán, M.; Körös, E. J. Phys. Chem. 1978, 82, 1672.

(12) Orbán, M.; Körös, E.; Noyes, R. M. J. Phys. Chem. 1979, 83, 3056.

(13) Orbán, M. J. Am. Chem. Soc. 1980, 102, 4311.

(14) Zykov, V. S. Simulation of Wave Processes in Excitable Media; Manchester University Press: Manchester, England, 1988.

(15) Zhabotinsky, A. M.; Müller, S. C.; Hess, B. Chem. Phys. Lett. 1990, 172,445 .
(16) Zhabotinsky, A. M.; Müller, S. C.; Hess, B. Physica D 1991, 49,

(17) Zhabotinsky, A. M.; Györgyi, L.; Dolnik, M.; Epstein, I. R. J. Phys. Chem. 1994, 98, 7981.

(18) Liu, J.; Scott, S. K. J. Chem. Soc., Faraday Trans. 1992, 88, 909.

(19) Giles, C. C. D.; Ibison, P.; Liu, J.; Scott, S. K. J. Chem. Soc., Faraday Trans. 1992, 88, 917.

(20) Györgyi, L.; Varga, M.; Körös, E.; Field, R. J.; Ruoff, P. J. Phys. Chem. 1989, 93, 2836.

(21) Kablaoni, M. S. J. Org. Chem. 1974, 39, 3696.

(22) Evans, J. C.; Klix, R. C.; Bach, R. D. J. Org. Chem. 1988, 53 5519.

(23) Musso, H.; Dopp, V. Ber. Deutsch. Chem. Ges. 1964, 97, 1147.

(24) Bugrim, A. E.; Zhabotinsky, A. M.; Epstein, I. R. J. Phys. Chem., in press.

JP953356J 